\title{
How Planetary Nebulae Shells Interact with their Local Environement
}

\author{
Eva Villaver ${ }^{1}$, Arturo Manchado ${ }^{2}$, Guillermo García-Segura ${ }^{3}$, and \\ Letizia Stanghellini ${ }^{4}$ \\ ${ }^{1}$ Space Telescope Science Institute, 3700 San Martin Drive, Baltimore MD 21218, USA; \\ Affiliated to the Hubble Space Telescope Department of ESA \\ ${ }^{2}$ Instituto de Astrofísica de Canarias, vía Láctea s/n, La Laguna, E-38200 Tenerife, Spain; \\ Affiliated to CSIC \\ ${ }^{3}$ Instituto de Astronomía-UNAM, Apartado postal 877, Ensenada, 22800 Baja California, \\ México \\ ${ }^{4}$ National Optical Astronomy Observatory, 950 N Cherry Avenue, Tucson AZ 85719, USA
}

\begin{abstract}
Planetary Nebula (PN) shells and AGB circumstellar envelopes evolve under a wide range of external conditions, from the high ISM densities found in the Galactic plane, to the rarefied and hot intracluster medium where the systemic velocity of the star can be as high as $2000 \mathrm{~km} \mathrm{~s}^{-1}$. We explore the effects that the external pressure and/or stellar systemic velocity have on the observable properties of PNe. We investigate how the mass and size of the PN halos are reduced when the star is moving with respect to the external medium. We have studied as well how the mass of the circumstellar envelope is fed by ISM material when high ISM densities characteristic of the Galactic plane are considered. By studying the evolution of $\mathrm{PNe}$ in the intracluster medium in Virgo, we infer shorter PN lifetimes than what is usually adopted. This has important implications, since the assumed PN lifetime strongly affects the fraction of intracluster light derived from PN studies.
\end{abstract}

Keywords. hydrodynamics,stars: AGB and post-AGB, planetary nebulae: general, intergalactic medium

\section{Introduction}

Planetary Nebulae (PNe), typically observed through their bright [O III] $\lambda 5007 \AA$ emission line, are among the most useful tracers of stellar populations. Given their ubiquity and easy detection, PNe have been used to study stellar populations in our Galaxy and the Local Group and as probes of the intracluster stellar light stripped from galaxies.

The PN formation process itself is relatively well understood since the seminal work of Kwok et al. (1978). Therefore the focus in the recent years has been in understanding the processes that may cause the departure from spherical symmetry (stellar rotation, magnetized winds, accretion disks and binaries). As models become more sophisticated in trying to pin down the fine details of the PN formation process, little attention has been paid to PN evolution, which ultimately determines their detectability under different environments.

PN evolution is linked to the evolution of the star which ultimately depends mostly on the stellar initial mass. As the star determines the rate at which the energy is injected into the nebular shells, the environment sets the external pressure conditions. Moreover, stars often move subject to an external gravitational potential, causing ram stripping to peel the PN shells on timescales that depend on the external ram pressure. In the last few years, we have identified and attempted to quantify the relative importance of all these processes on the evolution of the PN shells. 


\section{Evolution at Rest: the Influence of the Initial Stellar Mass}

As stars die they inject momentum and energy into the ISM in the form of chemically enriched winds. Low- and intermediate-mass stars (1-8 $\left.\mathrm{M}_{\odot}\right)$ lose up to $80 \%$ of their initial mass during the Asymptotic Giant Branch (AGB). Heavy mass-loss is the signature of the late AGB phase: at uncertain rates of $10^{-6}$ to $10^{-3} \mathrm{M}_{\odot} \mathrm{yr}^{-1}$, on timescales of $\sim 10^{5}$ $10^{6} \mathrm{yr}$, it removes up to $7 \mathrm{M}_{\odot}$ of stellar material (Vassiliadis \& Wood 1993, Blöcker 1995).

Villaver, García-Segura \& Manchado (2002a) used the Vassiliadis \& Wood (1993) predictions for the mass-loss rate behavior during the AGB to explore the circumstellar gas structure for different initial masses and external ISM conditions. We allowed our grids to be large enough to follow the full stellar ejecta and quantified the effect of the external ISM pressure. The presence of the ISM provides a non-negligible source of pressure that for a factor of 10 increase in pressure decelerates (a factor of $\sim 1.5$ ) and compresses (density increases a factor of $\sim 0.2$ ) the external shells.

The gas structure resulting from the previous AGB evolution for each initial mass was used as the starting configuration by Villaver, Manchado \& García-Segura (2002b). Then we followed the PN formation by using post-AGB tracks from Vassiliadis \& Wood (1994) that comprise the mass range of PN progenitors. Our numerical models predict that the AGB mass-loss rate history should be observable in the form of large (up to $2.5 \mathrm{pc}$ ) low surface brightness shells (called halos) during the PN phase.

Halos carry important information about the mass-loss history during the AGB phase, as their size directly reflect the timescales of the envelope ejection. Shorter timescales directly imply larger halo sizes at lower AGB mass-loss rates. PN halos have $\mathrm{H} \alpha$ emissivities between 10 and 5000 times fainter than the main shell. According to our models, observations of PNe severely underestimate the ionized mass present in PNe because they mostly observe the main shell. Most of the ionized mass in PNe is contained in the faint detached halos and goes undetected due to their low surface brightness.

By following the wind evolution we can highlight the relative importance of the dynamical effects of ionization on the shell's evolution. During the early stages of the PN evolution, the main shell is formed by the ionization front and is not driven by the hot bubble because the wind velocity is not high enough for an adiabatic shock to develop. This effect has a direct observational consequence on the interpretation of the observed kinematical ages. We find that for all the initial masses and for young PNe the kinematical ages overestimate the real central star age. Later on in the evolution, as the hot bubble develops, the measured kinematical ages underestimate the central star age but only for the low-mass progenitors.

According to our models a large fraction of the mass of the external shells (up to $~ 70 \%$ for the $1 \mathrm{M}_{\odot}$ stellar model) belongs to ISM matter that had been swept up by the stellar wind. This amount decreases according to the assumed density of the ISM and according to the progenitor mass; the higher the progenitor mass the lower the fraction of the ISM material residing in the shells.

\section{The Interaction with the ISM}

In the past, the PN-ISM interaction process was studied by considering the relative movement when the nebular shell was already formed. As a consequence, high relative velocities, high ISM densities or the presence of a magnetic field were necessary in order to explain the observed asymmetries in the external shells of PNe. Villaver, García-Segura \& Manchado (2003) demonstrated that this is not the case when the PN formation is followed by considering the evolution of the stellar wind from the AGB. Moreover, we 

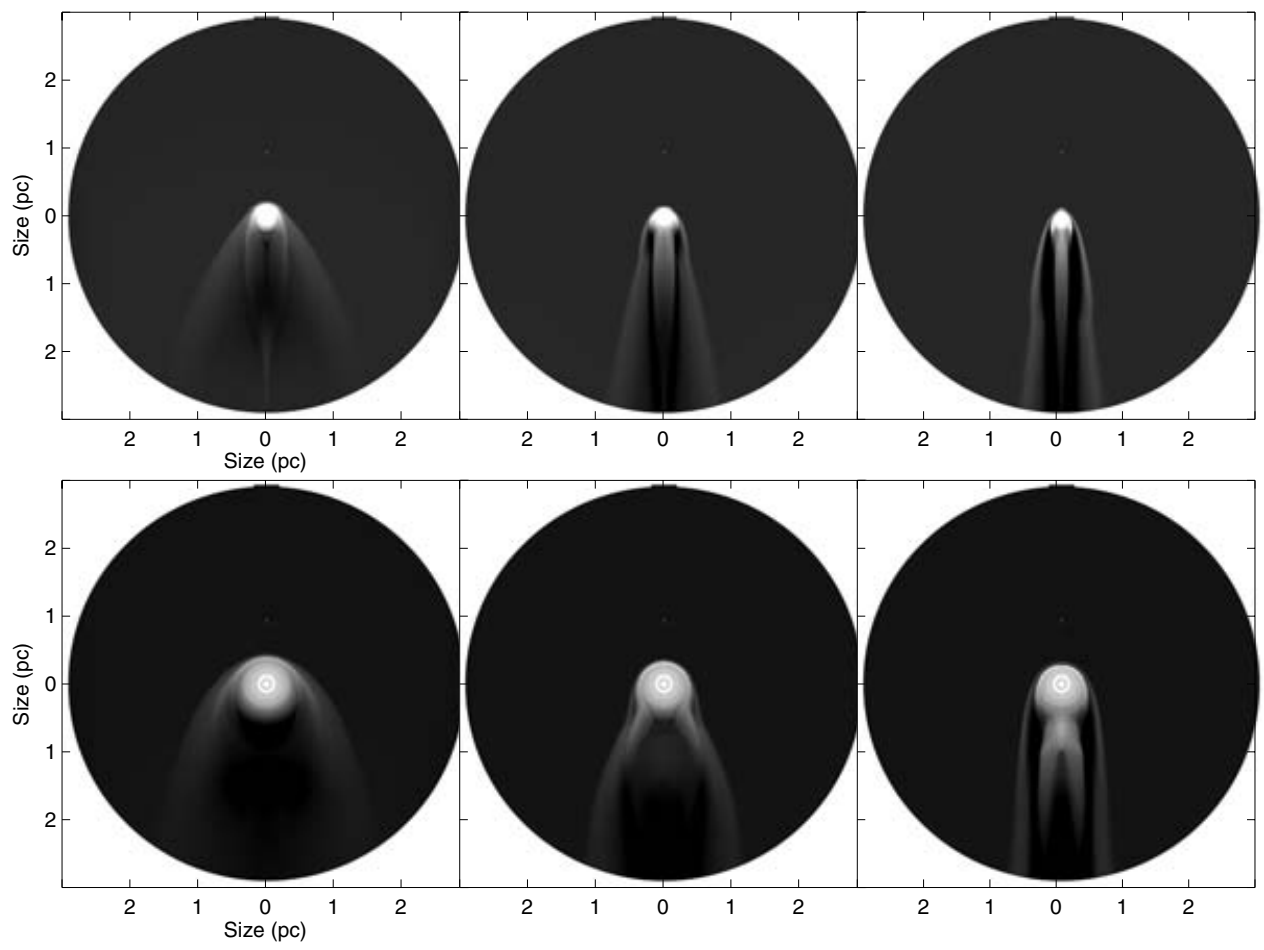

Figure 1. Snapshots of the (logarithmic) gas density generated during the AGB evolution of a $1 \mathrm{M}_{\odot}$ star moving through the ISM with different velocities. We show relative velocities of 20,50 and $100 \mathrm{~km} \mathrm{~s}^{-1}$ and an ISM density of $0.1 \mathrm{~cm}^{-3}$ with the ISM medium flowing into the grid from top to bottom. The evolution of the stellar wind during the thermal-pulsing AGB phase and the PN stage has been implemented at the center of the grid. The photoionization of the gas has also been considered. The three top snapshots have been taken at $2 \times 10^{5} \mathrm{yr}$ and have relative velocities of 20,50 and $100 \mathrm{~km} \mathrm{~s}^{-1}$ respectively, the same for the three bottom snapshots but taken $1.7 \times 10^{5}$ yr later.

show that the interaction is observable during the very early stages of the PN phase and it is prominent during the AGB evolution.

During the very early stages of the evolution of an AGB star the free-streaming steady stellar wind reaches ram pressure equilibrium with the ISM at a stand-off distance from the star that can be computed analytically. After this, the mass-loss rate and wind velocity change continuously in the inner boundary, giving rise to the formation of shocks that develop inside the bow-shock cavity formed by the early stationary wind. From then onwards the stand-off distance cannot be computed from a simple ram pressure balance argument because of the time-dependence of the AGB wind. The ram pressure of the ISM is balanced by the ram pressure of the stellar wind inside the bow-shock cavity, making it a time-dependent problem. We have explored the range of ram pressure conditions that a PN progenitor might experience by evolving in the Galaxy: from the high densities and low velocities in the plane to high velocities and rarefied medium in the halo (Villaver, García-Segura \& Manchado 2006, in prep.). In Figure 1 we show an example of how the morphology changes with the ram pressure considered. Note how the opening angle of the bow-shock changes with the relative velocity assumed. 


\section{PNe in the Intracluster medium}

Although PNe have been proved to be among the most useful tracers of intracluster (IC) light to date, no attempt has been made to explore the effects of the IC environment on PNe evolution. With this aim we have simulated the evolution of a $1 \mathrm{M}_{\odot}$ star as it moves with a velocity of $1000 \mathrm{~km} \mathrm{~s}^{-1}$ under the typical conditions observed in the Virgo IC medium. Villaver \& Stanghellini (2005) found that a PN evolving in the Virgo cluster has a different outer shell structure than that of a PN evolving in the Galaxy, but both PNe had similar observable properties when studied as point sources. The PN stripping process affects mainly the outer shell. However the bulk of the emission arises from the bright inner shell which dominates the emission when the PN is young. The main shell, for a given stellar mass, evolves similarly in the IC and in the Galaxy, and only gets affected by the ram pressure stripping process later on in the evolution, mostly when the PN has already fallen below observability limits.

The number of PNe in a given stellar population is usually computed assuming that the PN lifetime is $\sim 25000 \mathrm{yr}$. We find that a lifetime between 5000 and $10000 \mathrm{yr}$ should be used to predict the number of PNe per total luminosity in a given stellar population.

Finally work is in progress to describe the evolution of a fast moving star through a much higher density environment, such as that of the Leo I cloud, where no IC PNe were found. The higher ram pressure provided by this environment might remove the material from the inner PN shell, reducing the IC PN lifetime much further, thus preventing IC PN detection.

\section{Conclusions}

Given current prescriptions for stellar evolution, most of the stellar envelope ejected during the AGB should be observable in the form of large (up to $2.5 \mathrm{pc}$ ) low surface brightness shells during the PNe phase (Villaver et al. 2002a,b). However, we have shown how ram pressure stripping reduces both the size and the mass of this envelope (Villaver et al. 2003). Even a small relative velocity between the star and the external medium plays an important role in the evolution of the PN shell. Work is in progress that shows how a larger ram pressure than that considered for the Galactic models has catastrophic effects for the PN lifetime.

Finally we have explored the evolution of a PN under the extreme conditions found in the IC medium in Virgo (Villaver \& Stanghellini 2005). We confirm the PN survival and we find that the evolution of a $\mathrm{PN}$ in the IC medium in Virgo is different from a galactic model in the details of the outer shell structure, but they have similar observable properties when studied as point sources.

\section{References}

Bloecker, T. 1995, A\&A, 297, 727

Kwok, S., Purton, C. R., \& Fitzgerald, P. M. 1978, ApJ 219, L125

Vassiliadis, E. \& Wood, P. 1993, ApJ 413, 641

Vassiliadis, E. \& Wood, P. 1994, ApJ 92, 125

Villaver, E., García-Segura, G. \& Manchado, A. 2002a, ApJ 571, 880

Villaver, E., Manchado, A. \& García-Segura, G. 2002b, ApJ 581, 1204

Villaver, E., García-Segura, G. \& Manchado, A. 2003, ApJ 585, L49

Villaver, E. \& Stanghellini, L. 2005, ApJ 632, 854 